\title{
Human papillomavirus infection and p53 protein expression in vulvar intraepithelial neoplasia and invasive squamous cell carcinoma
}

D.E.S. Engelman,

L.A.L.A. Andrade and J. Vassallo
Departamento de Patologia Anatômica, Faculdade de Ciências Médicas, Universidade Estadual de Campinas, Campinas, SP, Brasil
Correspondence

D.E.S. Engelman

Avenida Princesa D'Oeste, 1180

Apto 42

13095-010 Campinas, SP

Brasil

Fax: +55-19-3232-7567

E-mail: diana@mpcnet.com.br

Publication supported by FAPESP.

P....

Received September 9, 2002 Accepted May 7, 2003

\begin{abstract}
The etiopathogenesis of vulvar intraepithelial neoplasia (VIN III) and invasive squamous cell carcinoma are largely unknown. Since there are few studies on Brazilian patients, our purpose was to determine the frequency of human papillomavirus (HPV) infection and the expression of p53 in these lesions, and associate them with other factors such as age, morphological subtypes, multicentric and multifocal disease. Thirty-eight cases of VIN III, nine of superficially invasive carcinoma, and 55 of invasive vulvar carcinoma were retrospectively evaluated from 1983 to 1995 for the presence of HPV by immunohistochemistry and in situ hybridization, and for p53 protein expression by immunohistochemistry on paraffin sections. All cases for whom material (slides and paraffin blocks) and clinical data were available were included. HPV and p53 were detected in 57.9 and $21.1 \%$ of the VIN III lesions, 33.3 and $66.7 \%$ of superficially invasive carcinomas, and 7.3 and $58.2 \%$ of invasive squamous cell carcinomas, respectively. HPV infection was associated with younger age in the VIN III and invasive carcinoma groups. In the latter, HPV infection was associated with the basaloid variant. p53 expression rate was higher in superficially invasive and invasive lesions and was not related to HPV infection. Our findings are similar to others and support the hypothesis that there are two separate entities of the disease, one associated with HPV and the other unrelated, with p53 inactivation possibly being implicated in some of the cases.
\end{abstract}

\section{Introduction}

Several lines of evidence have shown the involvement of human papillomavirus (HPV) in cervical carcinogenesis (1-4). Its oncogenic role has been attributed to a transforming capacity of the oncogenic types $16 / 18$, encoding two transforming gene products,
Key words

- Vulvar intraepithelial

neoplasia

- Vulvar carcinoma

- Human papillomavirus

- p53 protein the E6 and E7 oncoproteins. One of the mechanisms described is the interaction of E6 with the tumor suppressor gene p53 product, leading to loss of its normal function and thus to impaired cell proliferation and reparative function, increased susceptibility to mutations, and malignant transformation (2-7). 
A number of epidemiological studies have linked vulvar squamous cell carcinoma to some of the risk factors described for cervical carcinoma, and it has been shown that a proportion of the former are associated with HPV infection $(8,9)$. It seems that HPV is related to a minority of cases, especially in younger women, with the basaloid variant of squamous carcinoma and associated vulvar intraepithelial neoplasia (VIN) $(1,2,9,10)$. HPV DNA is detected in about $10-20 \%$ of all vulvar squamous cell carcinomas $(6,11,12)$, and in $50-80 \%$ of the basaloid subtype of invasive carcinoma $(2,5,8,11)$. However, most vulvar carcinomas occur in older patients, are predominantly of the usual keratinizing type and are associated with lichen sclerosus and squamous hyperplasia, suggesting that vulvar carcinomas may have different etiologies, with HPV and other factors possibly playing a significant role and are still a matter of debate $(1,2,9,10-14)$.

Current data point to the p53 gene as the leading factor in the surveillance of DNA integrity. Inactivation of its tumor suppressing activity seems to be an almost universal step in the development of human malignancies and may also be related to tumor progression $(2,4,6,7)$.

p53 inactivation in vulvar carcinomas can occur either by interaction with HPV E6 oncoprotein, or by p53 gene mutation in the absence of the virus. In addition, several cellular proteins and events can also interfere with or inhibit p53-mediated transactivation functions (2-7,13). p53 expression has been described in 50 to $88 \%$ of cases of vulvar squamous cell carcinoma $(2,5,6,15-18)$.

The purpose of the current study was to determine the frequency of HPV infection and p53 expression in primary VIN and invasive squamous cell carcinoma in Brazilian patients and to associate them with other factors such as age and morphologic subtypes. Since there are few studies on vulvar neoplasia in Brazil, our intention was to compare our findings with those for the worldwide population described in the literature, and to contribute additional cases.

\section{Material and Methods}

In a retrospective study conducted from 1983 to 1995,38 cases of VIN grade III, nine of superficially invasive carcinoma, and 55 of invasive squamous cell carcinoma of the vulva were collected and reviewed from the files of the Department of Pathology of the State University of Campinas.

Biopsies were taken from areas found to be altered upon physical examination or that were positive for the Collins test. Our selection was based on the most representative fragment of the lesion, the presence of histological signs consistent with HPV infection, and the presence of altered and normal epithelium adjacent to the neoplasia.

Multiple neoplastic lesions in the vulvar region separated by non-neoplastic epithelium were considered to be multifocal disease. The presence of intraepithelial neoplasia or invasive carcinoma at other sites of the lower female genital tract such as the vagina and uterine cervix was considered to be multicentric disease. Superficially invasive carcinoma was defined as stromal invasion of $1 \mathrm{~mm}$ or less (19).

Patient age, morphological type of the neoplasia, the presence of multifocal and multicentric lesions, and the epithelial alterations adjacent to the neoplasia were analyzed and correlated with HPV detection and p53 expression.

New sections of paraffin-embedded specimens were placed on sylanized slides for immunohistochemistry and in situ hybridization. The specimens were deparaffinized and rehydrated.

Immunohistochemistry was performed for HPV and p53 protein detection respectively using the anti-HPV polyclonal antibody (Dakopatts, Carpinteria, CA, USA) diluted 1:150, and the monoclonal antibody clone DO-7 (Dakopatts) diluted 1:100, which 
detects both the wild-type and mutant forms of the p53 protein, after inhibition of endogenous peroxidase with $3 \%$ hydrogen peroxidase in methanol solution. Antigen retrieval was performed by heating with a household microwave oven (2 cycles of 5 min each at $750 \mathrm{~W}$ for HPV detection and 3 cycles of 7 min each at $750 \mathrm{~W}$ for $\mathrm{p} 53$ ) in $10 \mathrm{mM}$ citrate buffer, $\mathrm{pH}$ 6.0. After rinsing with PBS solution, the slides were exposed to a biotinylated secondary antibody (polyclonal, 1:200; Multilink, Dako, Carpinteria, CA, USA). The reaction was visualized by the streptavidinbiotin-peroxidase method (Dakopatts). The slides were stained with diaminobenzidine substrate solution and counterstained with Mayer's hematoxylin.

In situ hybridization was performed with biotinylated probes for HPV types 6/11, 16/ 18, and 31/35/51 (Dakopatts), after digestion with a $0.08 \%(\mathrm{w} / \mathrm{v})$ pepsin solution (Sigma, St. Louis, MO, USA) at $37^{\circ} \mathrm{C}$ for 10 min. After overnight incubation of tissue with the probes at $37^{\circ} \mathrm{C}$ and rinsing with stringent solution (1:50, Dakopatts), the reaction was visualized using the streptavidinbiotin-peroxidase detection system (CSA System, Dakopatts). The slides were stained with diaminobenzidine substrate solution and counterstained with Mayer's hematoxylin.

Positive and negative controls were included in each protocol. Immunostaining for p53 protein was assessed by a semiquantitative method, considering the number of positive cells and their intensity within the negative epithelium, and graded as follows: - (negative), + (less than $10.0 \%$ of positive cells), ++ (10.0\% or more, and less than $50.0 \%$ of positive cells), and $+++(50.0 \%$ or more of positive cells) $(17,19,20)$. At least 200 cells were analyzed in each case. HPV was considered to be absent (-) or present (+) regardless of the number of positive nuclei for both methods.

Statistical analysis was performed using the Statistical Analysis System software package (SAS Institute Inc., Cary, NC, USA) and
"EpiInfo, 6.02b" (Centers for Disease Control and Prevention, Atlanta, GA, USA) and one of the following tests when appropriate: chi-square test to analyze the association between the variables in the three groups of carcinomas, Fisher exact test when the expected value was less than five, and Student $t$-test when comparing ages. Univariate and multivariate logistic regression analysis was performed to verify which of the variables analyzed were associated with HPV detection and $\mathrm{p} 53$ expression. A P value of 0.05 or less was considered to be statistically significant.

The present study was approved by the Research Ethics Committee of the Faculty of Medical Sciences, State University of Campinas.

\section{Results}

\section{Vulvar intraepithelial neoplasia}

This group included 38 patients with a mean age $( \pm$ SD) of $47.5 \pm 19.9$ years. Multicentric disease (macroscopic) was present in $36.8 \%$ of the cases and multifocal lesions (microscopic) in $34.2 \%$. Histological subtypes were mostly warty $(92.0 \%)$ and basaloid in only $8.0 \%$. In $42.1 \%$ of the cases there was an association with HPV-induced lesions and in $6.0 \%$ with squamous hyperplasia. HPV was detected by one of the methods in 22 of 38 cases (57.9\%), and p53 expression in $21.1 \%$, all of them graded as (+), i.e., with less than $10.0 \%$ of positive cells. HPV type $6 / 11$ alone was present in one case, and HPV $16 / 18$ in 16 cases, three of them associated with HPV 6/11 and one with HPV 31/ 35/51. In five cases, HPV was detected only by immunohistochemistry. The presence of HPV infection was associated with younger patient age (40.8 vs 57.3 years, $\mathrm{P}=$ $0.02)$. p53 expression was not related to any of the parameters analyzed, such as age, HPV detection and histological subtype of VIN III. 


\section{Invasive squamous cell carcinoma}

This group included 55 patients with a mean age of $67.8 \pm 12.5$ years. Multicentric disease was present in $7.3 \%$ and multifocal lesions in $23.6 \%$ of the cases. Histological subtypes were: $78.2 \%$ usual keratinizing, $10.9 \%$ basaloid, $7.3 \%$ warty and $3.6 \%$ verrucous. Associated epithelial changes corresponded mostly to dystrophic lesions (60.0\%), but VIN III (25.5\%) and HPV-associated lesions $(7.3 \%)$ were also present. The remaining cases $(7.2 \%)$ contained normal adjacent epithelium. Inguinal nodal metastases were present in $56.8 \%$ of the cases and in $16.4 \%$, the disease was recurrent. HPV capsid antigen was detected in three cases and HPV DNA in two. Overall, HPV was detected by one of the methods in 4 of 55 cases (7.3\%), all of them HPV type 16/18. p53 was expressed in 58.2\% of the cases, being graded as ++/+++ in $47.4 \%$. HPV detection was associated with younger age, presence of other neoplastic lesions in the female lower genital tract, and presence of the basaloid subtype of invasive carcinoma. However, by logistic regression analysis, only age (46 vs 69.5 years, $\mathrm{P}=0.05$ ) and the presence of multicentric disease $(\mathrm{P}=0.04)$ remained significant. p53 expression was only related to

Table 1. Clinicopathological findings of patients with three types of lesions.

\begin{tabular}{lccc}
\hline Variables & $\begin{array}{c}\text { VIN III } \\
(N=38)\end{array}$ & $\begin{array}{c}\text { SIC } \\
(N=9)\end{array}$ & $\begin{array}{c}\text { Invasive carcinoma } \\
(\mathrm{N}=55)\end{array}$ \\
\hline Age (mean \pm SD; years) & $47.5 \pm 19.9$ & $57.9 \pm 16.6$ & $67.8 \pm 12.5$ \\
Multicentric disease & $14(36.8)$ & $5(55.6)$ & $4(7.3)$ \\
Multifocal lesions & $13(34.2)$ & $5(55.6)$ & $13(23.6)$ \\
HPVAL & $16(42.1)$ & $2(22.2)$ & $4(7.3)$ \\
HPV capsid antigen & $13(34.2)$ & $3(33.3)$ & $3(5.5)$ \\
HPV DNA & $17(44.7)$ & $2(22.2)$ & $2(3.6)$ \\
HPV antigen or DNA & $22(57.9)$ & $3(33.3)$ & $4(7.3)$ \\
p53 expression & $8(21.1)$ & $6(66.7)$ & $32(58.2)$ \\
\hline
\end{tabular}

HPV: human papillomavirus; HPVAL: lesions with histological changes consistent with HPV infection (HPV-associated lesions); SIC: superficially invasive carcinoma of the vulva; VIN III: vulvar intraepithelial neoplasia grade III. The numbers within parentheses are percent. The invasive carcinoma group was statistically different from VIN III and SIC groups, which were similar except for p53 expression. p53 expression was similar for the invasive carcinoma and SIC groups and significantly different from the VIN III group. $P<0.001$, chi-square test for proportions. depth of invasion $(\mathrm{P}=0.04)$. Patients with the basaloid subtype of invasive carcinoma were younger (mean age, 56.8 years), and this subtype was associated with VIN III in $83.3 \%$ and with dystrophic lesions in the adjacent epithelium in $16.7 \%$. HPV was present in $50.0 \%$ and p53 in $33.3 \%$ of the cases. For the usual keratinizing type, mean age was 68.9 years, and this subtype was associated with VIN III in $20.9 \%$ and with dystrophic lesions in $60.5 \%$. HPV was detected in $2.3 \%$ and $\mathrm{p} 53$ in $60.5 \%$ of these cases. In five patients (11.6\%) there was no abnormality in the adjacent epithelium and in three (7.0\%), no adjacent epithelium was available for evaluation.

Lymph node metastasis was associated with involvement of the clitoris $(\mathrm{P}=0.005)$, tendency to a tumor size larger than $2 \mathrm{~cm}$ $(\mathrm{P}=0.05)$, and grade of the neoplasia $(\mathrm{P}=$ $0.05)$.

When more than one exam for the same patient was available in our files we compared the presence of relapse in the invasive vulvar carcinoma group. Relapse was associated with younger mean age $(54.6 \pm 13.8 v s$ $70.3 \pm 10.5$ years, $\mathrm{P}=0.0002$ ) and was not related to the presence of multicentric and multifocal disease, or to HPV detection and p53 expression.

\section{Superficially invasive carcinoma}

The mean age of the nine patients with superficially invasive carcinoma was $57.9 \pm$ 16.6 years. Multicentric disease was present in five cases and multifocal lesions in five. In eight cases, the warty type of VIN III was present in adjacent epithelium and HPVassociated lesions were present in two. HPV was detected in three cases (33.3\%) and p53 in six $(66.7 \%)$.

Table 1 summarizes the main clinicopathological data of the three groups of lesions.

\section{Discussion}

In agreement with other reports, we show 
that HPV infection associated with VIN III lesions occurred predominantly in younger patients, and was not related to p53 expression $(6,15,16,21)$.

In spite of having detected only nine patients with superficially invasive vulvar carcinomas between 1983 and 1995, our intention was to identify any similarity with the VIN III or with the invasive carcinoma group. Their clinicopathological characteristics resembled those of the VIN III group, except for the $\mathrm{p} 53$ protein, with a rate similar to that of the invasive carcinoma group. The association with VIN III in the adjacent epithelium in eight of nine superficially invasive vulvar carcinomas indicates that VIN III lesions may progress to invasive disease $(10,14,22)$

HPV infection is related to VIN III lesions and to a small subset of younger patients with invasive vulvar carcinoma of the basaloid variant, which is frequently associated with adjacent VIN III (1,2,9-11). Most patients with invasive carcinomas have the usual histological type, which is related neither to HPV infection nor to VIN III lesions, and is associated with higher rates of dystrophic lesions (1,2,9-14).

The finding of high rates of $\mathrm{p} 53$ expression in invasive carcinoma supports the role of p53 inactivation, either by mutation or by binding with HPV E6 oncoprotein (2-7). Nevertheless, as also reported elsewhere $(17,23)$, p53 expression was not related to HPV infection, being negative in 20 of the 55 cases and both (p53 and HPV) positive in only one case. This indicates that other factors besides HPV infection and p53 inactivation may be involved in the development of vulvar carcinoma $(1,2,9,11-13)$.

Our low rate of HPV and the finding of the oncogenic type $16 / 18$ in most of the positive cases were similar to that reported in the literature for vulvar carcinoma $(9,12)$. However, the rate was lower than in other studies in which a more sensitive technique (PCR) was used $(5,8,12)$. Our study was performed on formalin-fixed and paraffinembedded tissues. Although the more sensitive methods can be performed on this kind of specimens, DNA damage reduces their sensitivity. In our experience (data not shown), DNA extraction from paraffin-embedded tissues is successful in only a quarter of the cases, a fact that would reduce even more our series. In addition, topographic relations are lost using these methods. For this reason, in situ hybridization was adopted in the present study.

The condylomatous subtype of squamous cell carcinoma presented characteristics similar to those of the usual type and was not associated with the presence of HPV infection. This finding is in contrast to data reported for other series (9). However, this could be due to the small number of this carcinoma variant in our study, or to limitations of the method applied, i.e., in situ hybridization.

The low rate of $\mathrm{p} 53$ expression in VIN III lesions, and the higher rate in superficially invasive and invasive carcinoma suggest that p53 expression occurs as a late event in vulvar carcinogenesis, and is related to the capacity of stromal invasion by the neoplasia, as described for other sites (23). In our study, among the patients with invasive carcinoma, p53 detection was related only to the depth of invasion, and was not related to the presence of lymph node metastases or recurrent disease. These findings are similar to some studies (2) and contrast with others $(6,8,16)$.

VIN III is not a frequent condition and little is known about its natural history, but most studies have shown a low rate of progression to invasive disease of about $10.0 \%$ $(22,24)$. Studies of p53 in VIN lesions are rare, most of them associated with invasive carcinoma, and with contradictory results. Some have shown that $\mathrm{p} 53$ protein expression increased with the severity of the intraepithelial neoplasia, suggesting that p53 expression is related to the proliferation of 
abnormal cells $(13,14)$. Although more research is necessary, it seems that p53 might be useful as a progression marker for intraepithelial neoplastic lesions $(13,14)$.

In order to reduce surgical morbidity, to identify which patients will develop recurrent disease and metastasis, several prognostic markers have been proposed, including p53 $(12,25,26)$. There are also studies investigating its role as a predictor of radiotherapy and chemotherapy resistance $(7,25)$. However, the results are contradictory. Some reports associate the presence of $\mathrm{p} 53$ mutation or protein expression with a worse outcome, high rates of metastasis and advanced stage $(6,16)$, while others do not $(2,13,26)$, although the latter studies reported that p53 was associated with the disease-free interval. According to some reports, recurrent disease is not associated with HPV or p53 protein detection $(2,12,13,25,26)$. More research is needed to determine the role of $\mathrm{p} 53$ in the progression of the disease, as a prognostic marker and as a predictor of the tumor response to different treatments.

The characteristics of our patients are similar to those described in the literature and support the hypothesis that there are two separate entities of the disease, with HPV infection being responsible for the development of VIN III lesions and for a few cases of vulvar carcinomas $(1,2,9,11,12)$. On the other hand, p53 alterations may be implicated in the pathogenesis of most cases of invasive vulvar carcinomas $(5,6,13,15)$. However, $36.4 \%$ of the cases were negative for both HPV and p53, indicating that about onethird of the vulvar carcinomas might arise through a still unknown mechanism independent of p53 inactivation or HPV infection (12-14).

The number of cases in our study was higher than in several published reports. Until recently, vulvar studies were scarce and the etiology of vulvar carcinoma and its pathways are still a matter of debate. In addition, few vulvar studies are available in Brazil and our intention was to contribute additional cases, to compare our findings with published reports, and to stimulate further investigations on vulvar diseases.

\section{References}

1. Costa S, Syrjänen S, Vendra C, Chang F, Guida G, Hippeläinen M, Terzano P, Tervahauta A, Yliskoski M \& Syrjänen K (1995). Human papillomavirus infections in vulvar precancerous lesions and cancer. Journal of Reproductive Medicine, 40: 291-298.

2. Kagie MJ, Kenter GG, Tollenaar RAEM, Hermans J, Trimbos JB \& Fleuren GJ (1997). P53 protein overexpression is independent of human papillomavirus infection in squamous cell carcinoma of the vulva. Cancer, 80: 1228-1233.

3. Thomas M, Pim D \& Banks L (1999). The role of the E6-p53 interaction in the molecular pathogenesis of HPV. Oncogene, 18: 76907700.

4. Stoler MH (2000). Human papillomaviruses and cervical neoplasia: a model for carcinogenesis. International Journal of Gynecological Pathology, 19: 16-28.

5. Lee YY, Wilczynski SP, Chumakov A, Chih D \& Koffler UP (1994). Carcinoma of the vulva: HPV and p53 mutations. Oncogene, 9: 1655-1659.

6. Pilotti S, D'Amato L, Della Torre G, Donghi R, Langoni A, Giarola M, Sampietro G, De Palo G, Pierotti MA \& Rilke F (1995). Papillomavirus, p53 alteration, and primary carcinoma of the vulva. Diagnostic Molecular Pathology, 4: 239-248.

7. Velculescu VE \& El-Deiry WS (1996). Biological and clinical impor- tance of the p53 tumor suppressor gene. Clinical Chemistry, 42: 858-868.

8. Hørding U, Junge J, Daugaard S, Lundvall F, Poulsen H \& Bock JE (1994). Vulvar squamous cell carcinoma and papillomaviruses: indications for two different etiologies. Gynecologic Oncology, 52: 241246.

9. Trimble CL, Hildensheim A, Brinton LA, Shah KV \& Kurman RJ (1996). Heterogeneous etiology of squamous carcinoma of the vulva. Obstetrics and Gynecology, 87: 59-64.

10. ACOG Technical Bulletin (1994). Vulvar cancer. International Journal of Gynecology and Obstetrics, 44: 79-86.

11. Andersen WA, Franquemont DW, Williams J, Taylor PT \& Crum CP (1991). Vulvar squamous cell carcinoma and papillomaviruses: two separate entities? American Journal of Obstetrics and Gynecology, 165: 329-336.

12. Pinto AP, Lin MC, Mutter GL, Sun D, Villa LL \& Crum CP (1999), Allelic loss in human papillomavirus-positive and -negative vulvar squamous cell carcinomas. American Journal of Pathology, 154: 1009-1015.

13. Carlson JA, Amin S, Malfetano J et al. (2001). Concordant p53 and $\mathrm{mdm}-2$ protein expression in vulvar squamous cell carcinoma and adjacent lichen sclerosus. Applied Immunohistochemistry and Mo- 
lecular Morphology, 9: 150-163.

14. Yang B \& Hart WR (2000). Vulvar intraepithelial neoplasia of the simplex (differentiated) type. American Journal of Surgical Patholo$g y, 24: 429-441$

15. Hietanen S, Grénman S, Syrjänen K, Lappalainen K, Kauppinen J, Carey T \& Syrjänen S (1995). Human papillomavirus in vulvar and vaginal carcinoma cell lines. British Journal of Cancer, 72: 134-139.

16. Kohlberger PD, Kirnbauer R, Bancher D, Gitsch G, Reinthaller A, Leodolter S, Tschachler E, Kainz C \& Breitenecker G (1998). Absence of p53 protein overexpression in precancerous lesions of the vulva. Cancer, 82: 323-327.

17. Kim JW, Cho YH, Lee CG, Kim JH, Kim HK, Kim EJ, Han KT \& Namkoong SE (1997). Human papillomavirus infection and TP53 gene mutation in primary cervical carcinoma. Acta Oncologica, 36: 295-300.

18. Tervahauta Al, Syrjänen SM, Väyrynen M, Saastamoinen J \& Syrjänen KJ (1993). Expression of p53 protein related to the presence of human papillomavirus (HPV) DNA in genital carcinomas and precancer lesions. Anticancer Research, 13: 1107-1111.

19. Benedet JL, Bender H, Jones 3rd H, Ngan HY \& Pecorelli S (2000). FIGO staging classifications and clinical practice guidelines in the management of gynecologic cancers. FIGO Committee on Gynecologic Oncology. International Journal of Gynecology and Obstetrics, 70: 209-262

20. Vassallo J, Derchain SF, Pinto GA, Martinez EZ, Syriänen KJ \& Andrade LA (2000). High risk HPV and p53 protein expression in cervical intraepithelial neoplasia. International Journal of Gynecology and Obstetrics, 71: 45-48.

21. Edwards CL, Tortolero-Luna G, Linares AC, Malpica A, Baker WV Cook E, Johnson E \& Mitchell MF (1996). Vulvar intraepithelial neoplasia and vulvar cancer. Obstetrics and Gynecology Clinics of North America, 23: 295-324.

22. Herod JJO, Shafi MI, Rollason TP, Jordan JA \& Luesley DM (1996). Vulvar intraepithelial neoplasia with superficially invasive carcinoma of the vulva. British Journal of Obstetrics and Gynaecology, 103: 453-456.

23. Chetty R, Bramdev A, Aguirre-Arteta A, Pegoraro RJ \& Sataar N (1997). Relation between retinoblastoma and p53 proteins in human papilloma viruses $16 / 18$ positive and negative cancers of the uterine cervix. Journal of Clinical Pathology, 50: 413-416.

24. Junge J, Poulsen H, Horn T, Hørding U \& Lundvall F (1997). Prognosis of vulvar dysplasia and carcinoma in situ with special reference to histology and types of human papillomavirus (HPV). Acta Pathologica, Microbiologica et Immunologica Scandinavica, 105: 963-971.

25. Kagie MJ, Kenter GG, Tollenaar RAEM, Hermans J, Trimbos JB \& Fleuren GJ (1997). P53 protein overexpression, a frequent observation in squamous cell carcinoma of the vulva and in various synchronous vulvar epithelia, has no value as a prognostic parameter. International Journal of Gynecological Pathology, 16: 124-130.

26. McConnell DT, Miller ID, Parkin DE \& Murray GI (1997). P53 protein expression in a population-based series of primary vulval squamous cell carcinoma and immediate adjacent field change. Gynecologic Oncology, 67: 248-254. 